\section{ACRL Amendment Defeated in Council}

At the first meeting of the ACRL Board of Directors on Monday evening, June 21, the Committee on Academic Status made known its serious reservations about the proposed Program of Action of the ALA Staff Committee on Mediation, Arbitration and Inquiry. It moved that the Board support an amendment to the Program which would provide that the staff committee "shall not have jurisdiction over matters relating to the status and problems of academic librarians except on an interim basis," and that the interim should last only through August 31, 1972. It also stipulated that procedures be set up by ACRL to protect the rights of academic librarians. (For the full amendment see the minutes of the Board of Directors meetings in this issue.) The Board agreed the amendment should be brought before the ALA Council when the Program was proposed.

At the Council meeting on Friday, June 25, the program of action was presented. Mr. James Holly, ALA Councilor and member of the ACRL Board of Directors, moved the amendment which was seconded by Mr. E. J. Josey. On a roll call vote, the amendment was defeated 137 to 19 with two members answering "present."

At the Midwinter Meeting in Chicago in January 1970, the ALA Council voted that the results of all roll call votes be published. Following is the vote on the ACRL amendment.

Yeas-Lee Ash, Genevieve M. Casey, Richard M. Dougherty, Andrew J. Eaton, Anne C. Edmonds, Harold J. Erickson, James F. Holly, Andrew H. Horn, Ida-Marie Jensen, Arthur E. Jones, Sara Dowlin Jones, E. J. Josey, John P. McGowan, Arthur Monke, James W. Pirie, William Powell, William Roehrenbeck, Joseph H. Treyz, James $\mathrm{O}$. Wallace.

Nays-Elizabeth Abolin, Hugh C. Atkinson, Mary C. Baker, Mary C. Barter, R. Paul Bartolini, Helen H. Bennett, Lillian M. Bradshaw, James H. Burghardt, Edwin Castagna, Virginia Chase, Irene Christopher, Geraldine Clark, Jean D. Cochran, Betty Coughlin, Carolyn Crawford, Arthur Curley, Marie A. Davis, Richard DeGennaro, William DeJohn, Robert F. Delzell, William Dix, Keith Doms, Lawrence J. Downey, Kenneth F. Duchac, Elizabeth Edwards, Richard B. Engen, Lois Engler, John Fall, Alice Hild Farris, Paul J. Fasana, Elizabeth T. Fast, John D. Forsman, Donell J. Gaertner, Ervin J. Gaines, Janice Gallinger, Thomas J. Galvin, Donna Garcia, Charles A. Gardner, Mary V. Gaver, Andrew Geddes, William S. Geller, John Glinka, Emerson Greenaway, Pearce S. Grove, Mary Ann Hanna, Gustave A. Harrer, Frances S. Hatfield, William F. Hayes, William T. Hen- derson, David W. Heron, William Heuer, Peter Hiatt, Grace Hightower, Sr. Nora Hillery, Sam W. Hitt, Anna Hornak, Marie V. Hurley, James G. Igoe, Mrs. Alice Ihrig, Robert K. Johnson, H. G. Johnston, Virginia Lacy Jones, Mary Kahler, Frances Kennedy, Anne E. Kincaid, Margaret M. Kinney, Thelma Knerr, John C. Larsen, Mary E. Ledlie, Evelyn Levy, Joseph W. Lippincott, Helen Lockhart, John G. Lorenz, Jean E. Lowrie, Robert R. McClarren, Jane S. McClure, Stanley McElderry, Jane A. McGregor, Elizabeth B. Mann, Marion A. Milczewski, Eric Moon, Madel J. Morgan, Effie Lee Morris, Florrinell F. Morton, Margaret M. Mull, William D. Murphy, William C. Myers, Mrs. Karl Neal, Mildred L. Nickel, Eileen F. Noonan, Philip S. Ogilvie, A. Chapman Parsons, Richard Parsons, Anne Pellowski, Mary E. Phillips, Margaret E. Poarch, Patricia Pond, Gary R. Purcell, David L. Reich, Margaret G. Reid, Helen Renthal, John B. Richard, James H. Richards, Dorothy E. Rosen, Virginia L. Ross, Lelia B. Saunders, Catherine Schoenmann, Bella E. Shachtman, Brooke E. Sheldon, Marietta Daniels Shepard, Giles F. Shepherd, Shirley Shisler, Joseph F. Shubert, Sara L. Siebert, Marion L. Simmons, Clare M. Smith, Mildred K. Smock, C. Sumner Spalding, Mary Mace Spadling, Charles H. Stevens, Mary Ann Swanson, Franklin P. Taplin, Donald E. Thompson, Marguerite G. Thompson, Gertrude $R$. Thurow, June Thurston, Ralph A. Van Handel, Melvin J. Voigt, Ruth Waldrop, Myrna M. Wegner, Raymond E. Williams, Donald E. Wright, Barbara Westby (substitute), Helen T. Yast, Wayne Yenawine (substitute), Roberta E. Young, Willard $O$. Youngs.

Present-Page Ackerman, John A. Axam.

No Answer-Richard S. Angell, Mrs. Augusta Baker, Ralph H. Blackburn, James E. Bryan, William S. Budington, Sr. Mary Claudia Carlen, Marietta Chicorel, George W. Coen, C. Donald Cook, Essae Martha Culver, Richard L. Darling (presiding), Charles Dalrymple, Robert B. Downs, Leila Doyle, David E. Estes, Mrs. Loleta D. Fyan, Clarence R. Graham, Warren J. Haas, Dean Halliwell, David R. Hoffman, Mrs. Ruth Sheahan Howard, Edwin G. Jackson, Robert E. Lee, Milton E. Lord, Stephen A. McCarthy, Errett W. McDiarmid, Roger H. McDonough, Newman F. Mallon, Guy Marco, Lowell A. Martin, Keyes D. Metcalf, Foster E. Mohrhardt, Lucile M. Morsch, Orin M. Moyer, L. Quincy Mumford, Ralph Munn, William T. Peters, Benjamine E. Powell, Douglas G. Reid, John S. Richards, Carrie Robinson, Mary U. Rothrock, Frank B. Sessa, Ralph R. Shaw, Jessie Carney Smith, Frances Lander Spain, Basil Stuart-Stubbs, F. Charles Taylor, Kenneth Taylor, Betty Torricelli, Ralph A. Ulveling, Jane N. Vance, Allen B. Veaner, Carl Vitz, Robert Vosper, Frederick H. Wagman, Albert L. Williams, Louis Round Wilson. 


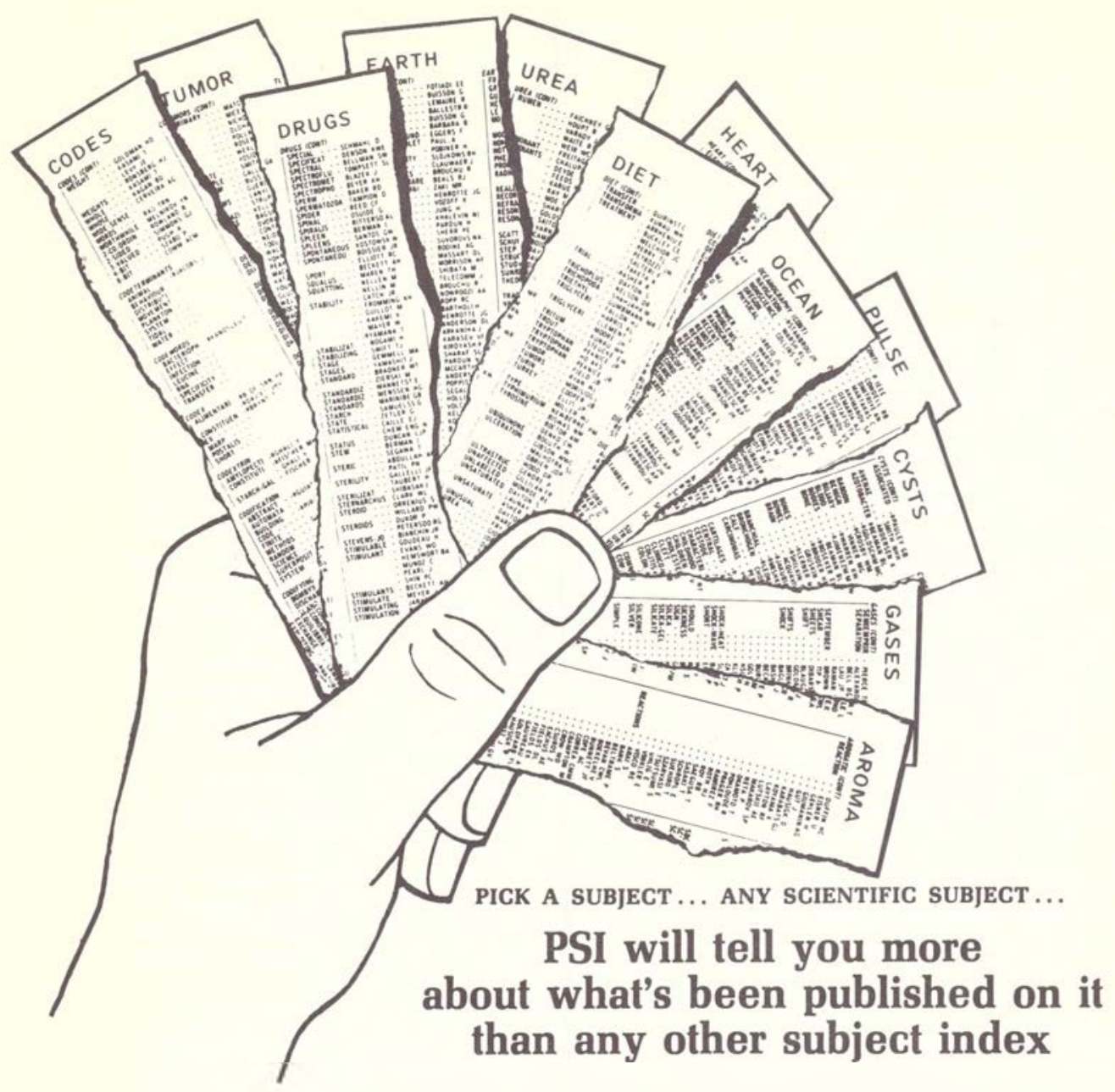

- Permuterm ${ }^{\circledR}$ Subject Index is the one guide to the world's current scientific and technical literature that is issued quarterly and annually and covers all disciplines, comprehensively, in easy-to-use natural language.

- The world's largest research libraries-libraries that have practically every major literature search tool published-find that the Permuterm Subject Index is the index of choice among experienced reference librarians and their patrons.

- PSI covers the entire field of published scientific literature-over 350,000 articles a year from over 2,400 key journals, in all disciplines, from all over the world. And it covers it in depth-every article and editorial item, of every issue.

- That's why smaller college, hospital, depart-

mental, and corporate libraries, too, find the Permuterm Subject Index one of their most valuable reference tools. It's the one subject index that finds the information they need no matter what the subject or discipline-no matter where the article was published.

- PSI literature searches are fast and easy, free of highly complex search techniques. Because PSI is arranged in easy-to-use format, with title words "permuted" or arranged in every possible two-word combination to form hundreds of access points to most topics.

- Be sure your library and its patrons enjoy the unique advantages of the Permuterm Subject Index, not only at a central location, but at branch and departmental libraries as well. For more on the PSI and what it can mean to you, send for the free PSI brochure. Today.

Institute for Scientific Information
Phila., Pa. 19106, Tel: (215) 923-3300 